\title{
Venous thromboembolic events in critically ill traumatic brain injury patients
}

\section{Skrifvars, Markus}

2017-03

Skrifvars , M , Bailey, M , Presneill , J French , C , Nichol , A , Little , L , Duranteau , J , Huet , O , Haddad , S , Arabi , Y , McArthur , C , Cooper , D J \& Bellomo , R 2017 , ' Venous thromboembolic events in critically ill traumatic brain injury patients ' , Intensive Care Medicine , vol. 43 , no. 3 , pp. 419-428 . https://doi.org/10.1007/s00134-016-4655-2

http://hdl.handle.net/10138/234133

https://doi.org/10.1007/s00134-016-4655-2

unspecified

publishedVersion

Downloaded from Helda, University of Helsinki institutional repository.

This is an electronic reprint of the original article.

This reprint may differ from the original in pagination and typographic detail.

Please cite the original version. 


\title{
ORIGINAL
}

\section{Venous thromboembolic events in critically ill traumatic brain injury patients}

Markus B. Skrifvars ${ }^{1,2^{*}}$, Michael Bailey ${ }^{1}$, Jeffrey Presneill ${ }^{1,3}$, Craig French ${ }^{4,5}$, Alistair Nichol ${ }^{1,6,7}$, Lorraine Little ${ }^{1}$, Jacques Duranteau ${ }^{8}$, Olivier Huet ${ }^{9}$, Samir Haddad ${ }^{10}$, Yaseen Arabi $^{10}$, Colin McArthur ${ }^{11}$, D. James Cooper ${ }^{1,7}$, Rinaldo Bellomo ${ }^{1,12}$ and For the EPO-TBI investigators and the ANZICS Clinical Trials Group

(C) 2016 Springer-Verlag Berlin Heidelberg and ESICM

\begin{abstract}
Purpose: To estimate the prevalence, risk factors, prophylactic treatment and impact on mortality for venous thromboembolism (VTE) in patients with moderate to severe traumatic brain injury (TBI) treated in the intensive care unit.

Methods: A post hoc analysis of the erythropoietin in traumatic brain injury (EPO-TBI) trial that included twiceweekly lower limb ultrasound screening. Venous thrombotic events were defined as ultrasound-proven proximal deep venous thrombosis (DVT) or clinically detected pulmonary embolism (PE). Results are reported as events, percentages or medians and interquartile range (IQR). Cox regression analysis was used to calculate adjusted hazard ratios (HR) with 95\% confidence intervals (Cl) for time to VTE and death.

Results: Of 603 patients, 119 (19.7\%) developed VTE, mostly comprising DVT (102 patients, 16.9\%) with a smaller number of PE events (24 patients, 4.0\%). Median time to DVT diagnosis was 6 days (IQR 2-11) and to PE diagnosis 6.5 days (IQR 2-16.5). Mechanical prophylaxis (MP) was used in $91 \%$ of patients on day 1, 97\% of patients on day 3 and $98 \%$ of patients on day 7 . Pharmacological prophylaxis was given in $5 \%$ of patients on day $1,30 \%$ of patients on day 3 and $57 \%$ of patients on day 7. Factors associated with time to VTE were age (HR per year 1.02, 95\% Cl 1.01-1.03), patient weight (HR per $\mathrm{kg} 1.01,95 \% \mathrm{Cl} 1-1.02$ ) and TBI severity according to the International Mission for Prognosis and Analysis of Clinical Trials risk of poor outcome (HR per 10\% increase 1.12, 95\% Cl 1.01-1.25). The development of VTE was not associated with mortality (HR 0.92, 95\% Cl 0.51-1.65).
\end{abstract}

Conclusions: Despite mechanical and pharmacological prophylaxis, VTE occurs in one out of every five patients with TBI treated in the ICU. Higher age, greater weight and greater severity of TBI increase the risk. The development of VTE was not associated with excess mortality.

Keywords: Erythropoietin, Deep venous thrombosis, Pulmonary embolism, Traumatic brain injury, Venous thromboembolism

\footnotetext{
*Correspondence: markus.skrifvars@monash.edu

${ }^{1}$ Australian and New Zealand Intensive Care Research Centre, School

of Public Health and Preventive Medicine, Monash University, 55

Commercial Rd, Melbourne, VIC 3004, Australia

Full author information is available at the end of the article
}

Take-home message: Despite mechanical and pharmacological prophylaxis, venous thromboembolism occurs in one of five patients with traumatic brain injury treated in the intensive care unit. Older age, greater weight and traumatic brain injury severity increase this risk.

\section{重 Springer}




\section{Introduction}

Traumatic brain injury (TBI) patients treated in the intensive care unit (ICU) appear at high risk for venous thromboembolism (VTE), such as deep venous thrombosis (DVT) of the legs and pulmonary embolism (PE) [13]. Moreover, the occurrence of massive PE is a known cause of late death in trauma patients, and the risk is increased in patients with DVT $[4,5]$. However reported VTE incidence rates vary considerably in the literature depending on whether the diagnosis was made using screening protocols or only by assessing symptomatic patients [5, 6]. Treatment options for preventing VTEs include mechanical prophylaxis (MP) and pharmacological prophylaxis (PP) [7]. MP is undertaken by means of compressive stockings or pneumatic compression devices and their use may be limited in trauma patients with severe injuries to the lower limbs. Pharmacological prophylaxis is undertaken using daily injections of either low molecular weight heparin (LMWH) or unfractionated heparin (UH) [7]. However, given the risk of aggravating intracranial bleeding in TBI patients the initiation of pharmacological prophylaxis is commonly delayed and later initiation may increase the risk of VTE [2]. Despite the above uncertainties about true incidence, risk of PErelated mortality and variability in the timing and extent of MP and PP application, there is a lack of data from prospectively screened large cohorts of TBI patients for the diagnosis of DVT with simultaneously collected data on the timing and use of MP and PP.

Between 2010 and 2014, we conducted the EPOTBI trial in 606 patients with moderate or severe TBI [8]. Because of the possible pro-thrombotic effects of erythropoietin (EPO), the study included twice-weekly screening leg ultrasounds (US) during ICU stay, enabling objective systematic assessment for DVTs [9-12]. In addition, the collected data included extensive evaluation of pre-injury risk as well as daily assessment of VTE risk factors and the use and type of prophylaxis. We have previously reported that the use of EPO did not increase the risk of VTE or other thrombotic events [8]. In the present study, we assessed the occurrence of VTE over time in the ICU and identified factors associated with the occurrence of VTE. We hypothesized that VTE would be more common in patients with a more severe TBI and that risk would be high in patients with delayed initiation of prophylaxis.

\section{Materials and methods}

The EPO-TBI trial was a multicentre, multinational, randomised, double-blind, parallel-group, placebo controlled trial that enrolled 606 patients with non-penetrating moderate [best post-resuscitation, pre-intubation Glasgow Coma Score (GCS) 9-12] or severe (GCS 3-8)
TBI. Patients were randomised to receive either weekly doses of 40,000 IU of epoetin alfa (Eprex Janssen-Cilag Pty Ltd, Titusville, NJ, USA) or placebo $(0.9 \%$ sodium chloride) up to three doses or until ICU discharge. Given the previously reported potential pro-thrombotic effects of EPO, the study excluded the following patients with a high risk of VTE: (1) haemoglobin (Hb) level above the upper limit of normal at each enrolling institution, (2) spinal cord injury, (3) history of DVT or PE, (4) known chronic hypercoagulable disorder (factor Leiden V, prothrombin G20210A, protein C deficiency, protein S deficiency and antithrombin III deficiency, patients 3 months postpartum and malignancy). In addition, patients at risk of arterial thrombosis such as chronic renal failure with an arteriovenous fistula in situ or myocardial infarction within the last 12 months were also excluded. The second and third doses of EPO were only given if the $\mathrm{Hb}$ did not exceed $120 \mathrm{~g} / \mathrm{L}$.

Patients were followed up extensively throughout ICU and hospital care. An electronic case report form included daily data on the use of blood transfusions, haematology results, the use of mechanical and pharmacological VTE prophylaxis and the performance of lower limb US examinations while in the ICU. The occurrence of DVT on ultrasound and other thrombotic events were predefined secondary outcomes of the study.

\section{Screening ultrasound}

Bilateral compression Doppler US of the lower limbs was performed in the ICU at baseline and twice weekly until ICU discharge or for 3 weeks in total, whichever occurred first. In cases of clinical suspicion of a DVT, further US were performed as per order of the treating clinician. Compression ultrasounds were performed by either accredited US technician or a radiologist according to local practice. Standard operating procedures for the performance of screening US and diagnosis of DVTs were used at all institutions. The US screening procedure focused on the deep veins of lower limbs above the knee i.e. (1) the trifurcation of the deep calf veins, (2) distal popliteal, (3) proximal popliteal, (4) distal femoral, (5) mid femoral and (6) common femoral. Compressibility of veins was tested in 1-cm increments, and non-compressibility was taken to indicate thrombosis. Thrombi occurring in the distal part of the lower limb such as in the saphenous vein were documented but not considered a DVT. All US were performed at the bedside.

\section{Diagnosis of pulmonary embolism}

Investigations for diagnosing or ruling out PE were defined by the treating clinician. The study protocol included guidelines on PE diagnosis and management. In brief, PE was recommended to be diagnosed with a spiral 
computed tomography (CT) scan of the lungs with intravenous contrast. The diagnosis of PE required the demonstration of an intraluminal filling defect of the main, lobar or segmental branches of the pulmonary artery. Alternative diagnostic methods included ventilation-perfusion scanning or pulmonary angiography. In cases of diagnosed PE US of the lower limbs were recommended as well. Possible PEs diagnosed at autopsy were not systematically sought and were not included.

\section{Use of mechanical or pharmacological prophylaxis}

The use of mechanical and pharmacological DVT prophylaxis was recommended and outlined in the study protocol according to the following principles: mechanical prophylaxis (MP) including anti-embolism compression stockings and/or pneumatic compression devices was recommended in all patients unless contraindicated. The use of an inferior vena cava filter could be considered in cases of large peripheral thrombi and the presence of an absolute contraindication to anticoagulation therapy. The recommended drug for PP was subcutaneous enoxaparin at $40 \mathrm{mg}$ daily. Timing was recommended to be based on the findings of the second brain CT scan and decided in liaison with the neurosurgical team. Prophylaxis was recommended to be continued on hospital wards after ICU discharge.

\section{Statistical analysis}

A statistical analysis plan was developed a priori and approved by all authors. Categorical data are presented as numbers and percentages and compared using Chisquare test. Numerical data are presented as medians and interquartile range (IQR) in parenthesis and compared using the Mann-Whitney $U$ test. Two separate Cox regression models were developed for time to death and time to occurrence of DVT with MP and PP treated as time-dependent variables. For the mortality model, patients were censored at 180-day follow-up, whilst for the DVT model, patients were censored at hospital discharge or death. We decide a priori to include factors found significant in univariate analysis as well as previously reported risk factors for VTE in the model. We included the following known factors for VTE: age [13], injury severity [14], severity of TBI [15] and the presence of pelvic fracture [16]. To further account for potential heterogeneity between hospitals, location was also included in the multivariable model as a random effect. Proportionality assumptions were confirmed using Schoenfeld residuals. As a further exploratory sensitivity analysis of the impact of pharmacological prophylaxis, we studied the development of VTE at various predefined time points (ICU days 3 and 7). For these cross-sectional analyses, we excluded patients that had developed a
VTE prior to this time point. We also tested whether the development of VTE was associated with 6-month mortality. This model included the same outcome covariates as specified in the main paper [17]. Associations between blood test values over time and the use of pharmacologic prophylaxis and the development of VTE were tested using repeated measures analysis of variance (ANOVA). Statistical analysis was performed with SPSS version 22.0 (IBM Corp. Released 2013. IBM SPSS Statistics for Windows, Version 22.0. Armonk, NY: IBM Corp.) and SAS version 9.4 (SAS Institute Inc., Cary, NC, USA).

\section{Results}

The EPO study screened a total of 3384 patients from May 2010 to November 2014 (Fig. 1). A total of 2778 were not included in the study: 2645 did not meet inclusion criteria and an exclusion related to risk of VTE or other thrombotic risk was present in 115 patients due to the inability to perform US, 93 due to an HB level exceeding the upper level of normal, 95 due to spinal injury, 17 due to pregnancy, 25 due to a history of DVT or PE, 17 due to a chronic hypercoagulable disorder, 7 due to acute myocardial infaraction (AMI) during the last 12 months and 2 due to chronic renal disease and dialysis (Fig. 1). Thus, the study included a total of 606 patients of whom three withdrew consent and were excluded from analysis.

\section{Screening ultrasounds and VTE}

A median of four screening ultrasounds were performed per patient (IQR 2-6). Of the 603 patients, 119 (19.7\%) developed VTE. Of these, 102 patients $(16.9 \%)$ had a DVT of the lower extremities and 24 patients (4.0\%) PE. Of these 119 patients, $95(80 \%)$ had only a diagnosis of DVT, 17 (14\%) only of PE and 7 (6\%) of both DVT and $\mathrm{PE}$. The median time to diagnosis from ICU admission to any form of VTE was 6 (2-12) days. The median time from ICU admission to diagnosis of DVT was 6 days (IQR 2-11); PE 6 days (IQR 2-17), and to a combined DVT and PE diagnosis within the same subject 7 days (IQR 2.5-13.5). In 14 of the 119 patients (12\%), VTE was diagnosed on the day of randomisation and in 35 patients (29.4\%) during the first 3 days. The development of VTE over time during the first 21 days in the ICU is shown in Fig. 2.

\section{Unadjusted assessment of risk factors for venous thrombotic events}

Baseline patient characteristics and prehospital treatment associations with the occurrence of a VTE are shown in Table 1. On unadjusted analysis, patients developing a VTE were older and had a higher body weight than those who did not develop a VTE. Gender was not related to the development of VTE in the whole cohort (Table 1), or 

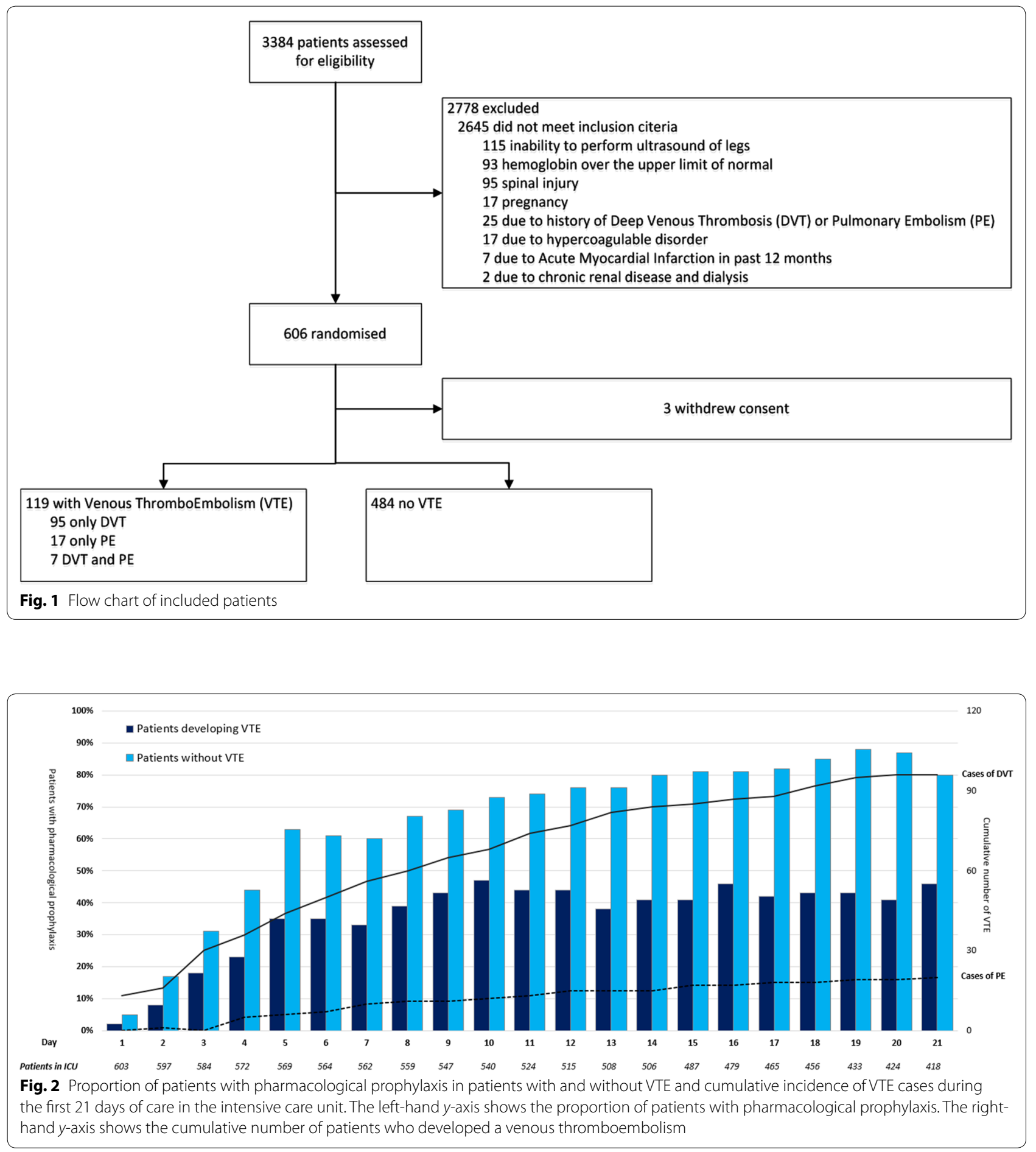

in a subset of patients under the age of 40 (377 patients) where 8 out of $49(16.3 \%)$ women developed a VTE compared to 44 out of $328(13.4 \%)(p=0.58)$. The unadjusted occurrence of VTE was also more common in Australia and New Zealand compared with other regions (Saudi
Arabia and Europe). The occurrence of VTE was more common in patients with more severe general injury as suggested by the injury severity score, more severe TBI as suggested by higher IMPACT probability of poor outcome at 6 months and more severe critical illness as 
Table 1 Demographic data including mechanism of injury and injury severity of included patients

\begin{tabular}{|c|c|c|c|c|}
\hline Characteristic & All patients $(n=603)$ & $\operatorname{VTE}(n=119)$ & No VTE $(n=484)$ & $p$ value \\
\hline Mean age (years) & $30.5(22.6-47.9)$ & $43.2(24.5-52)$ & $28.5(22.1-44.8)$ & $<0.0001$ \\
\hline Female gender & $101(17 \%)$ & $24(20 \%)$ & $77(16 \%)$ & 0.27 \\
\hline Weight (kg) & $75(65-86.5)$ & $80(72-92.5)$ & $75(65-85)$ & $<0.001$ \\
\hline \multicolumn{5}{|l|}{ Geographical region } \\
\hline Australia or New Zealand & $315(52.2 \%)$ & $78(24.8 \%)$ & $237(75.2 \%)$ & $<0.001$ \\
\hline Saudi Arabia or Europe & $288(47.8 \%)$ & $41(14.2 \%)$ & $247(85.8 \%)$ & \\
\hline \multicolumn{5}{|l|}{ Mechanism of injury } \\
\hline Motor vehicle accident & $237(39.3 \%)$ & $44(37.0 \%)$ & 193 (39.9\%) & 0.70 \\
\hline Motorcycle & $70(11.6 \%)$ & $13(10.9 \%)$ & $57(11.8 \%)$ & \\
\hline Bicycle & $26(4.3 \%)$ & $2(1.7 \%)$ & $24(5.0 \%)$ & \\
\hline Pedestrian & $58(9.6 \%)$ & $12(10.1 \%)$ & $46(9.5 \%)$ & \\
\hline Fall/jump & $138(22.9 \%)$ & $31(26.1 \%)$ & $107(22.1 \%)$ & \\
\hline Hit by object & $51(8.5 \%)$ & $12(10.1 \%)$ & $39(8.1 \%)$ & \\
\hline Other & $23(3.8 \%)$ & $5(4.2 \%)$ & $18(3.7 \%)$ & \\
\hline \multicolumn{5}{|l|}{$\mathrm{TB}$ I severity } \\
\hline Moderate TBI (GCS 9-12) & $149(24.7 \%)$ & $25(21.0 \%)$ & $124(25.6 \%)$ & 0.3 \\
\hline Severe TBI (GCS 3-8) & $454(75.3 \%)$ & $94(79.0 \%)$ & $360(74.4 \%)$ & 0.3 \\
\hline Median GCS & $7(4-9)$ & $6(3-8)$ & $7(4-9)$ & 0.06 \\
\hline \multicolumn{5}{|l|}{ Prehospital care } \\
\hline Systolic blood pressure less than $90 \mathrm{mmHg}$ & $190(31.5 \%)$ & $47(39.5 \%)$ & $143(29.5 \%)$ & 0.036 \\
\hline Oxygen saturation less than $90 \%$ & $115(19.1 \%)$ & $38(31.9 \%)$ & $77(15.9 \%)$ & $<0.0001$ \\
\hline \multicolumn{5}{|l|}{ Severity of TBI, illness and trauma } \\
\hline IMPACT-TBI probability of poor outcome & $45.8 \%(27.0-66.0)$ & $52.7 \%(39.1-71.8)$ & $39.1 \%(27.0-59.5)$ & $<0.001$ \\
\hline APACHE II score & $19(15-25)$ & $21(16-16)$ & $19(15-24)$ & 0.009 \\
\hline \multicolumn{5}{|l|}{ Abbreviated injury score } \\
\hline Head & $4(3-5)$ & $4(4-5)$ & $4(3-5)$ & 0.44 \\
\hline Face & $0(0-2)$ & $0(0-2)$ & $0(0-2)$ & 0.35 \\
\hline Chest & $2(0-3)$ & $2(0-3)$ & $2(0-3)$ & 0.37 \\
\hline Abdomen/pelvis & $0(0-0)$ & $0(0-2)$ & $0(0-0)$ & 0.009 \\
\hline Extremity & $0(0-2)$ & $0(0-2)$ & $0(0-2)$ & 0.45 \\
\hline External & $0(0-1)$ & $1(0-1)$ & $1(0-1)$ & 0.006 \\
\hline Injury severity score & $26(19-33)$ & $29(22-34)$ & $25(18-33)$ & 0.004 \\
\hline
\end{tabular}

suggested by higher APACHE II scores (Table 1). Specific known risk factors for VTE are shown in Table 2. On unadjusted analysis, the presence of a pelvic fracture was significantly associated with the occurrence of a VTE, whereas family history of VTE and smoking were not. The transfusion of red cells or fresh frozen plasma prior to randomisation was more common in patients that developed a VTE (Table 2). In addition, initial platelet levels in ICU were lower in patients who developed VTE (Table 2).

\section{Occurrence of VTE and use of mechanical and pharmacological prophylaxis}

A total of 453 (75.1\%) patients received some form of pharmaceutical prophylaxis (PP) during ICU stay and overall, when assessed in isolation, such treatment was not different in patients who developed VTE compared to those who did not (71 vs. $76 \%, p=0.30)$. In the unadjusted analysis, delay in initiation of such prophylaxis was longer in those who developed a VTE (median 6, IQR 3-10) compared to those who did not (median 4, IQR 3-6) $(p<0.001)$ (Supplementary Table 4). By day 3, 181 (30\%) patients had received PP, and this proportion was lower in those who developed a VTE compared to those who did not (20 vs. $32 \%, p=0.009$ ) (Supplementary Table 4). By day 7, 343 (57\%) patients had received $\mathrm{PP}$, and this proportion was lower in patients who developed a VTE compared to those who did not (43 vs. $60 \%$, $p=0.001$ ) (Supplementary Table 4). The use of PP over time in those patients who developed VTE and those that 
Table 2 Differences in patients with and without a venous thrombotic event

\begin{tabular}{|c|c|c|c|c|}
\hline Characteristic & All patients $(n=603)$ & $\operatorname{VTE}(n=119)$ & No VTE $(n=484)$ & $p$ value \\
\hline \multicolumn{5}{|l|}{ Risk factors } \\
\hline Family history of VTE & $7(1.2 \%)$ & $3(2.5 \%)$ & $4(0.8 \%)$ & 0.12 \\
\hline Pelvic fracture & $74(12.3 \%)$ & $21(17.6 \%)$ & $53(11.0 \%)$ & 0.046 \\
\hline Femoral fracture & $37(6.1 \%)$ & $9(7.6 \%)$ & $28(5.8 \%)$ & 0.47 \\
\hline Oestrogen & $12(2 \%)$ & $0(0 \%)$ & $12(2.5 \%)$ & 0.08 \\
\hline Significant immobility in past 3 months & $0(0 \%)$ & $0(0 \%)$ & $0(0 \%)$ & NA \\
\hline Smoker & $179(29.7 \%)$ & $32(26.9 \%)$ & $147(30.4 \%)$ & 0.46 \\
\hline None & $347(57.5 \%)$ & $65(54.6 \%)$ & $282(58.3 \%)$ & 0.47 \\
\hline \multicolumn{5}{|l|}{ Blood transfusions prior to randomisation } \\
\hline Red cells (\%) & $154(25.5 \%)$ & $41(34.5 \%)$ & $113(23.3 \%)$ & 0.013 \\
\hline Platelets (\%) & $57(9.5 \%)$ & $14(11.8 \%)$ & $43(8.9 \%)$ & 0.34 \\
\hline Fresh frozen plasma (\%) & $91(15.1 \%)$ & $29(24.4 \%)$ & $62(12.8 \%)$ & 0.002 \\
\hline Other clotting product (\%) & $45(7.5 \%)$ & $12(10.1 \%)$ & $33(6.8 \%)$ & 0.23 \\
\hline None (\%) & $422(70.0 \%)$ & $74(62.2 \%)$ & $348(71.9 \%)$ & 0.038 \\
\hline \multicolumn{5}{|l|}{ Previous medication } \\
\hline Unfractionated heparin & $0 \%$ & $0 \%$ & $0 \%$ & NA \\
\hline Low molecular weight heparin & $1(0.2 \%)$ & $0(0 \%)$ & $1(0.2 \%)$ & 0.62 \\
\hline Warfarin & $2(0.3 \%)$ & $1(0.8 \%)$ & $1(0.2 \%)$ & 0.28 \\
\hline Aspirin & $10(1.7 \%)$ & $2(1.7 \%)$ & $8(1.7 \%)$ & 0.98 \\
\hline Other & $1(0.2 \%)$ & $0(0 \%)$ & $1(0.2 \%)$ & 0.62 \\
\hline \multicolumn{5}{|l|}{ Initial haematological parameters } \\
\hline Haemoglobin & $121(104-134)$ & $120(101-136)$ & $121.5(104-133.5)$ & 0.53 \\
\hline INR & $1.2(1.1-1.2)$ & $1.1(1.1-1.2)$ & $1.2(1.1-1.25)$ & 0.49 \\
\hline APTT & $29.8(27-32.6)$ & $29.2(27-33)$ & $29.8(27-32.4)$ & 0.72 \\
\hline Platelets & $194(156-239)$ & $185(148-223)$ & $198(163-241)$ & 0.012 \\
\hline
\end{tabular}

did not is shown in Fig. 2. There were unadjusted regional differences in the initiation of PP with median delay to initiation in Australia and New Zealand of 5 days (IQR 3-9) compared to 4 days (IQR 2-5) in Europe and 4 days (IQR 3-6) in Saudi Arabia $(p<0.001)$. There was no difference in mean haemoglobin levels over time based on whether the patient received PP or not (95 vs. $95 \mathrm{~g} / \mathrm{l}$, $p=0.86$ ). In addition, there was no difference in mean activated thromboplastin time (APTT) values over time and the use of PP (32 vs. $31.9 \mathrm{~s} p=0.478$ ). There were no significant differences in the use of mechanical prophylaxis at day 1, 3 or 7 in patients who developed a VTE and those who did not (Supplementary Table 4).

\section{Blood transfusion and blood values and VTE}

In patients who developed a VTE after day 3 the median volume of red cells transfused prior to day 3 was $520 \mathrm{ml}$ (IQR 471-797 ml) compared to $560 \mathrm{ml}$ (IQR 300$815 \mathrm{ml})$ in those who did not develop a VTE $(p=0.30)$. In patients who developed a VTE after day 7 the median volume of red cells transfused prior to day 7 was $523 \mathrm{ml}$ (IQR 470-849) and $600 \mathrm{ml}$ (IQR $500-1000 \mathrm{ml}$ ) in those who did not $(p=0.53)$. Blood transfusion prior to day 3 or 7 did not increase the risk of developing a VTE at a later stage (Supplementary Tables 6, 7). The mean laboratory values of haemoglobin, international normalized ratio (INR), platelet count, and APTT and the administration of red blood cells, platelets, fresh frozen plasma and clotting products are shown in the electric supplementary material in Supplementary Figs. 3-6. There was no correlation between levels of haemoglobin $(p=0.67)$, platelets $(p=0.56)$, INR $(p=0.74)$ and VTE. There was no difference between VTE patents and those without with regards to transfusion of red cells (44\% compared to $37 \%, p=0.21)$, platelets $(7.6 \%$ compared to $8.3 \%$, $p=0.80$ ), fresh frozen plasma ( $10 \%$ compared to $8.3 \%$, $p=0.53)$ or other clotting products $(2.5 \%$ compared to $1.7 \%, p=0.53$ ) as shown in Supplementary Figs. 3-6.

\section{Multivariate modelling for development of VTE}

At ICU admission, independent predictors for the development of VTE were age (HR 1.02, 95\% CI 1-1.03, $p=0.01$ ), weight (HR 1.01, 95\% CI 1-1.02, $p=0.011$ ) and severity of TBI according to the IMPACT risk of poor outcome (HR 1.12, 95\% CI 1.01-1.25, $p=0.030$ ) (Supplementary Table 5). Factors associated with time to development 
Table 3 Cox regression model for factors associated with VTE

\begin{tabular}{lllll}
\hline Outcome & Univariate $\mathrm{HR}(95 \% \mathrm{Cl})$ & $p$ value & Multivariate OR (95\% Cl) & $p$ value \\
\hline Age (years) & $1.02(1.01-1.03)$ & 0.001 & $1.02(1-1.03)$ & 0.010 \\
Weight $(\mathrm{kg})$ & $1.02(1.01-1.03)$ & 0.002 & $1.01(1-1.02)$ & 0.011 \\
Region Australia and New Zealand & $2.12(1.27-3.53)$ & 0.004 & $2.09(0.99-4.4)$ & 0.053 \\
Region Europe & $0.77(0.39-1.53)$ & 0.460 & $0.66(0.28-1.57)$ & 0.35 \\
No family history of VTE & $0.72(0.1-5.14)$ & 0.740 & $1.09(0.15-8.01)$ & 0.93 \\
No pelvic fracture & $0.64(0.39-1.06)$ & 0.081 & $0.64(0.37-1.1)$ & 0.11 \\
Early transfusion of red cells or FFP & $0.78(0.52-1.17)$ & 0.230 & $1.15(0.73-1.84)$ & 0.54 \\
Injury severity score & $1.02(1-1.04)$ & 0.056 & $1(0.98-1.02)$ & 0.83 \\
IMPACT risk of poor outcome (in 10\%) & $1.15(1.05-1.25)$ & 0.002 & $1.12(1.01-1.25)$ & 0.03 \\
APACHE II score & $1.02(0.99-1.05)$ & 0.133 & $0.97(0.93-1)$ & 0.08 \\
Intervention (placebo vs. EPO) & $1.17(0.79-1.72)$ & 0.438 & $1.45(0.97-2.16)$ & 0.07 \\
Use of pharmacological prophylaxis (days) & $0.65(0.4-1.04)$ & 0.069 & $0.75(0.46-1.24)$ & 0.27 \\
\hline
\end{tabular}

of VTE in the ICU (excluding those patients with VTE present at the baseline screening ultrasound) with Cox regression are shown in Table 3 . The use and time to pharmacological prophylaxis was not independently associated with the development of VTE (Table 3). In a further exploratory analysis, the absence of PP on day 3 (HR 2.3, 95\% CI $0.98-5.47, p=0.06$ ) or day 7 (HR 1.72, 95\% CI 0.86-3.44, $p=0.13$ ) was not independently associated with VTE development thereafter (Supplementary Tables 6, 7).

\section{Occurrence of VTE and outcome}

Patients who developed VTE had significantly longer ICU and hospital stays, at 17 (IQR 11-22) and 33 (IQR 20-56) days compared to $12(6-19)$ and 23 (13-40) days, respectively ( $p<0.001$ for both). Median time on mechanical ventilation was longer in patients who developed VTE compared to those who did not, 11 days (IQR $6-17$ ) compared to 8 (IQR $4-14$ ), $p<0.001$. There was no difference in ICU (89.1 vs. $90.5 \%)$, hospital (88.2 vs. $88.4 \%$ ) or 6 -month survival ( 86.6 vs. $87.2 \%$ ) but good neurological outcome was less common in those who developed a VTE (45.4 vs. 57.9\%, $p=0.01$ ). Survival curves for patients alive at 7 days and at ICU discharge who had developed a VTE prior to that time point are shown in the Supplementary Fig. 7. In a multivariate model including age, presence of hypotension or hypoxia, presence of an intracranial mass lesion, pupils of equal size and reacting to light, study treatment EPO/placebo, the development of VTE was not associated with mortality (HR 0.81, 95\% CI 0.45-1.45) (Supplementary Table 8).

\section{Factors associated with occurrence of pulmonary embolism}

Patient who developed a PE were older than those who did not (49.5 IQR 24.5-58.5 vs. 30.3 IQR 22.5-46.9, $p=0.01$ ) (Supplementary Table 9). Pulmonary embolism was diagnosed more commonly in Australia and New Zealand than in Europe and Saudi Arabia (5.7 vs. 2.1\%, $p=0.02$ ). There was no difference in TBI severity, critical illness or injury severity (Supplementary Table 9). There was no difference in either risk factors, blood transfusions, admission haematological parameters or previous medication use in patients who developed PE and those who did not (Supplementary Table 10).

\section{Discussion}

Key findings

In this post hoc analysis of a multicentre international randomised controlled trial with prospective screening for DVT by means of compression leg ultrasound, we found that VTE occurs in approximately one out of every five patients with TBI, with almost a third occurring in the first 3 days. Moreover, we found that injury severity, age and weight are the three major independent risk factors for VTE, while delay in the use of mechanical prophylaxis was not found to be an independent predictor of VTE. Pharmacologic prophylaxis is commonly delayed and its impact on the development of VTE is unclear. Finally, VTE was more common in patients with prolonged ICU length of stay but was not independently associated with increased mortality.

\section{Relationship with previous studies}

The occurrence of VTE including proximal DVT was common in the present study and much higher than reported in a recent review of all studies on TBI patients, which found a reported VTE rate of $2.8 \%$ in over 4000 cases [2]. Interestingly our rate of proximal DVT is similar to rates found in the 1990s by Geerts et al. in a landmark study of 349 patients with major trauma [6], where 
venography was used for screening and a proximal DVT rate of $18 \%$ was found. Our results are also comparable to those of the recent study by Robertson and colleagues on TBI patients [11]. This suggests that there has been no major change in VTE rates in trauma patients over the last $20-30$ years.

The rate of both DVTs and PE is about four times greater in TBI patients than in general medical and surgical ICU patients [18]. The PROTECT study included over 4000 critically ill patients but excluded patients with major trauma. It compared the use of dalteparin or unfractionated heparin for the prevention of VTE [18]. It included an identical ultrasound screening protocol as in our study and found a 5.5\% rate of DVT and a $1.8 \%$ rate of PE. The reasons for the higher incidence of VTE in TBI patients are likely multifactorial and may include fractures of the pelvis and lower extremity and, perhaps, delays in initiation of PP. Indeed, studies have shown the use of inappropriate pharmacologic prophylaxis in up to $70 \%$ patients with major trauma [19].

The optimal timing for the initiation of PP in TBI patients has, however, not been defined [7]. In the current study, we found a clear association between later initiation of PP and the development of VTE in univariate analysis. However, this association was lost with multivariate modelling. This suggests that PP delays may simply be more common in patients with severe TBI who are independently prone to VTE. The point estimate of the hazard ratios does, however, suggest a potential but limited protective benefit. A lack of statistical power may have contributed to this finding.

Our findings that age and weight are important risk factors for the development of VTE are consistent with previous literature [20]. The age-related effect has been attributed to increases in various coagulation factors [21], increased tendency to platelet aggregation [22], impaired fibrinolysis [23] and pathophysiologic changes in the vasculature [24]. Previous studies have also linked obesity with the development of VTE [19]. This may be related to venous stasis [25] and a pro-thrombotic state [26]. It is also possible that the doses of PP used in the current study in heavier patients were too low. Indeed studies have suggested that weight-adjusted dosing of PP is important in order to achieve therapeutic levels of antifactor Xa [27]. Dosing of PP guided by anti-Xa measurements has been shown to reduce the incidence of DVT without increasing bleeding rates [28].

In the recent study by Robertson and colleagues on the use of a haemoglobin threshold of $100 \mathrm{~g} / \mathrm{l}$ in TBI patients for blood transfusion resulted in a higher prevalence of VTE [11]. In the current study we did not find any clear association with either haemoglobin or coagulation parameters and transfusion of red blood cells or other blood products, and the development of VTE. Regarding PE there were much less profound differences in patients developing PE and those who did not. This makes selective prophylactic strategies aiming at specifically reducing the risk of PE in high-risk patients difficult to implement. One such intervention with uncertain indications is the prophylactic placement of vena cava filters [29].

\section{Implications of study findings}

Our findings imply that in a population of patients with moderate to severe TBI one out of every five patients may develop either DVT or PE. Moreover, our findings imply that greater injury severity, older age and overweight should trigger consideration for particularly active surveillance and earlier and more aggressive protective measures whenever possible. Future studies should explore whether screening measures and earlier initiation of pharmacological prophylaxis are safe and effective in critically ill TBI patients. Finally, the observation that close to one-third of VTE events occur in the first 3 days implies that such surveillance should start as soon as possible after ICU admission.

\section{Study strengths and limitations}

This study has several strengths. It is the largest study to date of TBI patients to include a robust screening protocol for DVTs with regular lower limb ultrasonography. Its double-blind randomised controlled design allowed a clear understanding of the effect of EPO on the risk of DVT and VTE and provided a high level of internal validity. Its multicentre and multinational features provide a high degree of external validity. The collection of detailed daily data on VTE prophylaxis provides the first detailed insight into the current preventive management of VTE in TBI patients. However, our study also carries some limitations. Because of the possible prothrombotic effects of EPO, certain subgroups of patients were excluded, creating a selection bias. Thus, the overall true VTE incidence in unselected TBI patients might be even higher then reported in our study. It is noteworthy that some of the patients were excluded because of the inability to perform lower limb US. This may represent a patient group with both a high risk of DVT and a need for other screening strategies than US. In addition, our study case report form did not include patient height and thus we were unable to calculate body mass index which might have shown an even stronger relationship with VTE risk.

We also did not collect data on time of mobilisation and active infection or inflammation, which could contribute to the development of VTE. In addition, given the multicentre design of the study several different approaches to pharmacological prophylaxis were used, which may 
have influenced the occurrence of VTE [30]. Moreover, although we linked delayed PP with increased risk of VTE, we cannot confirm if such increased risk reflects the delayed PP per se or whether both delayed PP and increased risk reflect injury severity not fully captured by the illness severity scores used in our study.

\section{Conclusions}

In this large multicentre trial one in five patients with traumatic brain injury developed VTE despite rapid and near complete use of mechanical prophylaxis. This observed rate of VTE is four times higher than observed in other critically ill patients, with one-third of events developing within the first 3 days. Moreover, injury severity, older age and increasing weight are key independent risk factors for VTE.

\section{Electronic supplementary material}

The online version of this article (doi:10.1007/s00134-016-4655-2) contains supplementary material, which is available to authorized users.

\begin{abstract}
Author details
${ }^{1}$ Australian and New Zealand Intensive Care Research Centre, School of Public Health and Preventive Medicine, Monash University, 55 Commercial Rd, Melbourne, VIC 3004, Australia. ${ }^{2}$ Division of Intensive Care, Department of Anaesthesiology, Intensive Care and Pain Medicine, Helsinki University and Helsinki University Hospital, Helsinki, Finland. ${ }^{3}$ Department of Intensive Care, Royal Melbourne Hospital, Melbourne, VIC, Australia. ${ }^{4}$ Department of Intensive Care, Western Health, Melbourne, VIC, Australia. ${ }^{5}$ University of Melbourne, Melbourne, VIC, Australia. ${ }^{6}$ School of Medicine and Medical Sciences, University College Dublin, Dublin, Ireland. ${ }^{7}$ Department of Intensive Care and Hyperbaric Medicine, The Alfred, Melbourne, VIC, Australia. ${ }^{8}$ Département d'Anesthésie-Réanimation, Hôpital de Bicêtre, Assistance Publique des Hopitaux de Paris, Hôpitaux Universitaires Paris-Sud, Université Paris-Sud, Paris, France. ${ }^{9}$ Department of Anaesthesiology and Intensive Care Medicine, CHRU La Cavale Blanche, Université de Bretagne Occidentale, Brest, France. ${ }^{10} \mathrm{King}$ Saud Bin Abdulaziz University for Health Sciences and King Abdullah International Medical Research Center, Riyadh, Saudi Arabia. ${ }^{11}$ Department of Critical Care Medicine, Auckland City Hospital, Auckland, New Zealand. ${ }^{12}$ Department of Intensive Care, Austin Health, Melbourne, VIC, Australia.
\end{abstract}

\section{Acknowledgements}

Markus Skrifvars has received unrestricted grant support from Finska Läkaresällskapet, Medicinska Understödsföreningen Liv och Hälsa and Svenska Kulturfonden.

\section{Compliance with ethical standards}

\section{Conflicts of interest}

Markus Skrifvars reports having received a research grant from GE Healthcare and travel reimbursements and lecture fees from Orion Pharma, COVIDIEN, Astellas Pharma and Axis-Shield. All other authors report that they have no conflicts of interest.

Received: 1 November 2016 Accepted: 16 December 2016 Published online: 27 December 2016

\section{References}

1. Phelan HA (2013) Venous thromboembolism after traumatic brain injury. Semin Thromb Hemost 39:541-548

2. Abdel-Aziz H, Dunham CM, Malik RJ, Hileman BM (2015) Timing for deep vein thrombosis chemoprophylaxis in traumatic brain injury: an evidence-based review. Crit Care 19:96
3. Lier H, Bottiger BW, Hinkelbein J, Krep H, Bernhard M (2011) Coagulation management in multiple trauma: a systematic review. Intensive Care Med 37:572-582

4. Ho KM, Burrell M, Rao S, Baker R (2010) Incidence and risk factors for fatal pulmonary embolism after major trauma: a nested cohort study. Br J Anaesth 105:596-602

5. Allen CJ, Murray CR, Meizoso JP, Ginzburg E, Schulman Cl, Lineen EB, Namias N, Proctor KG (2016) Surveillance and early management of deep vein thrombosis decreases rate of pulmonary embolism in high-risk trauma patients. J Am Coll Surg 222:65-72

6. Geerts WH, Code Kl, Jay RM, Chen E, Szalai JP (1994) A prospective study of venous thromboembolism after major trauma. N Engl J Med 331:1601-1606

7. Brain Trauma Foundation, American Association of Neurological Surgeons, Congress of Neurological Surgeons, Joint Section on Neurotrauma and Critical Care, AANS/CNS, Bratton SL, Chestnut RM, Ghajar J, McConnell Hammond FF, Harris OA, Hartl R, Manley GT, Nemecek A, Newell DW, Rosenthal G, Schouten J, Shutter L, Timmons SD, Ullman JS, Videtta W, Wilberger JE, Wright DW (2007) Guidelines for the management of severe traumatic brain injury. V. Deep vein thrombosis prophylaxis. J Neurotrauma 24(Suppl 1):S32-S36

8. Nichol A, French C, Little L, Haddad S, Presneill J, Arabi Y, Bailey M, Cooper DJ, Duranteau J, Huet O, Mak A, McArthur C, Pettila V, Skrifvars M, Vallance S, Varma D, Wills J, Bellomo R, EPO-TBI Investigators, ANZICS Clinical Trials Group (2015) Erythropoietin in traumatic brain injury (EPO-TBI): a doubleblind randomised controlled trial. Lancet 386:2499-2506

9. Nichol A, French C, Little L, Presneill J, Cooper DJ, Haddad S, Duranteau J, Huet O, Skrifvars M, Arabi Y, Bellomo R, EPO-TBI Investigators and the Australian and New Zealand Intensive Care Society Clinical Trials Group (2015) Erythropoietin in traumatic brain injury: study protocol for a randomised controlled trial. Trials 16:39

10. Corwin HL, Gettinger A, Fabian TC, May A, Pearl RG, Heard S, An R, Bowers PJ, Burton P, Klausner MA, Corwin MJ, EPO Critical Care Trials Group (2007) Efficacy and safety of epoetin alfa in critically ill patients. N Engl J Med 357:965-976

11. Robertson CS, Hannay HJ, Yamal JM, Gopinath S, Goodman JC, Tilley BC, Epo Severe TBITI, Baldwin A, Rivera Lara L, Saucedo-Crespo H, Ahmed O, Sadasivan S, Ponce L, Cruz-Navarro J, Shahin H, Aisiku IP, Doshi P, Valadka A, Neipert L, Waguspack JM, Rubin ML, Benoit JS, Swank P (2014) Effect of erythropoietin and transfusion threshold on neurological recovery after traumatic brain injury: a randomized clinical trial. JAMA 312:36-47

12. Singh AK, Szczech L, Tang KL, Barnhart H, Sapp S, Wolfson M, Reddan D, Investigators C (2006) Correction of anemia with epoetin alfa in chronic kidney disease. N Engl J Med 355:2085-2098

13. Ekeh AP, Dominguez KM, Markert RJ, McCarthy MC (2010) Incidence and risk factors for deep venous thrombosis after moderate and severe brain injury. J Trauma 68:912-915

14. Ortel TL (2010) Acquired thrombotic risk factors in the critical care setting. Crit Care Med 38:S43-S50

15. Reiff DA, Haricharan RN, Bullington NM, Griffin RL, McGwin G Jr, Rue LW 3rd (2009) Traumatic brain injury is associated with the development of deep vein thrombosis independent of pharmacological prophylaxis. J Trauma 66:1436-1440

16. Knudson MM, Ikossi DG, Khaw L, Morabito D, Speetzen LS (2004) Thromboembolism after trauma: an analysis of 1602 episodes from the American College of Surgeons National Trauma Data Bank. Ann Surg 240:490-496 (discussion 496-498)

17. Presneill J, Little L, Nichol A, French C, Cooper DJ, Haddad S, Duranteau J, Huet O, Skrifvars M, Arabi Y, Bellomo R, EPO-TBI Investigators, ANZICS Clinical Trials Group (2014) Statistical analysis plan for the Erythropoietin in Traumatic Brain Injury trial: a randomised controlled trial of erythropoietin versus placebo in moderate and severe traumatic brain injury. Trials 15:501

18. PROTECT Investigators for the Canadian Critical Care Trials Group and the Australian and New Zealand Intensive Care Society Clinical Trials Group, Cook D, Meade M et al (2011) Dalteparin versus unfractionated heparin in critically ill patients. N Engl J Med 364:1305-1314

19. Garcia-Olivares P, Guerrero JE, Galdos P, Carriedo D, Murillo F, Rivera A (2014) PROF-ETEV study: prophylaxis of venous thromboembolic disease in critical care units in Spain. Intensive Care Med 40:1698-1708 
20. Ageno W, Becattini C, Brighton T, Selby R, Kamphuisen PW (2008) Cardiovascular risk factors and venous thromboembolism: a meta-analysis. Circulation 117:93-102

21. Tracy RP (2002) Hemostatic and inflammatory markers as risk factors for coronary disease in the elderly. Am J Geriatr Cardiol 11(93-100):107

22. Kasjanovova D, Adameckova D, Gratzlova J, Hegyi L (1993) Sex-related and age-related differences in platelet function in vitro: influence of hematocrit. Mech Ageing Dev 71:103-109

23. Gleerup G, Winther K (1995) The effect of ageing on platelet function and fibrinolytic activity. Angiology 46:715-718

24. Taddei S, Virdis A, Ghiadoni L, Salvetti G, Bernini G, Magagna A, Salvetti A (2001) Age-related reduction of NO availability and oxidative stress in humans. Hypertension 38:274-279 (Dallas, Tex: 1979)

25. Martinelli I, Bucciarelli P, Mannucci PM (2010) Thrombotic risk factors: basic pathophysiology. Crit Care Med 38:S3-S9

26. Franchini M, Targher G, Montagnana M, Lippi G (2008) The metabolic syndrome and the risk of arterial and venous thrombosis. Thromb Res 122:727-735
27. Nunez JM, Becher RD, Rebo GJ, Farrah JP, Borgerding EM, Stirparo JJ, Lauer C, Kilgo P, Miller PR (2015) Prospective evaluation of weight-based prophylactic enoxaparin dosing in critically III trauma patients: adequacy of antixa levels is improved. Am Surg 81:605-609

28. Ko A, Harada MY, Barmparas G, Chung K, Mason R, Yim DA, Dhillon N, Margulies DR, Gewertz BL, Ley EJ (2016) Association between enoxaparin dosage adjusted by anti-factor Xa trough level and clinically evident venous thromboembolism after trauma. JAMA Surg 151:1006-1013

29. Aryafar H, Kinney TB (2010) Optional inferior vena cava filters in the trauma patient. Semin Intervent Radiol 27:68-80

30. Beitland S, Sandven I, Kjærvik LK, Sandset PM, Sunde K, Eken T (2015) Thromboprophylaxis with low molecular weight heparin versus unfractionated heparin in intensive care patients: a systematic review with meta-anlaysis and trial sequential analysis. Intensive Care Med 41:1209-1219 